\title{
Severe Microcephaly Induced by Blockade of Vasoactive Intestinal Peptide Function in the Primitive Neuroepithelium of the Mouse
}

\author{
Pierre Gressens, ${ }^{\star \ddagger}$ Joanna M. Hill, ${ }^{\mathbf{f}}$ Benedicte Paindaveine," Illana Gozes,' Mati Fridkin, ${ }^{\star \star}$ and Douglas E. Brenneman ${ }^{\mathbf{1}}$ \\ * Laboratory of Experimental Neuropathology, National Institute of Neurological Disorders and Stroke, ${ }^{8}$ Section on Developmental and \\ Molecular Pharmacology, National Institute of Child Health and Human Development, and "Developmental Endocrinology Branch, \\ National Institute of Child Health and Human Development, National Institutes of Health, Bethesda, MD, 20892; ${ }^{\ddagger}$ Service of Pediatric \\ Neurology, University of Louvain Medical School, Brussels, Belgium; 'Department of Chemical Pathology, Tel Aviv University, \\ 69978 Tel Aviv, Israel; and ** Organic Chemistry Department, Weizmann Institute of Science, 76100 Rehovot, Israel
}

\begin{abstract}
Vasoactive intestinal peptide (VIP) has potent growth-related actions that influence cell mitosis, neuronal survival, and neurodifferentiation in cell culture. VIP can also produce dramatic growth in postimplantation mouse embryos in vitro, characterized by large increases in cell number. The goal of the present study was to assess the role of VIP on early nervous system development in vivo. Pregnant mice were treated with a specific antagonist to VIP. Prenatal administration of the antagonist early in development (E9. E11) produced severe microcephaly characterized by decreased embryonic brain weight with reduced DNA and protein content. The retardation of growth was disproportionally manifested in the brain compared with the body and was prevented by co-treatment with VIP. Identical treatment with the antagonist later in gestation had no detectable effect on embryonic growth. VIP receptors, which were restricted to the central nervous system during this stage of embryonic development, were increased in the neuroepithelium of antagonist-treated embryos while the number of cells in S-phase was significantly decreased. Thus, VIP regulates brain growth in vivo and inhibition of its action provides new insight into a molecular mechanism for microcephaly. (J. Clin. Invest. 1994. 94:2020-2027.) Key words: mitosis - growth retardation - embryo - growth factor $\cdot$ vasoactive intestinal peptide antagonist
\end{abstract}

\section{Introduction}

Understanding the pathophysiology of microcephaly is not only important for the development of preventive and therapeutic strategies, but also for identification of the essential elements

Address correspondence to Douglas E. Brenneman Ph.D., Section on Developmental and Molecular Pharmacology, Laboratory of Developmental Neurobiology, NICHD/NIH, Bldg. 49, Rm. 5A38, 9000 Rockville Pike, Bethesda, MD 20892.

Received for publication 22 March 1994 and in revised form 17 June 1994.

1. Abbreviations used in this paper: BRDU, bromodeoxyuridine; CNS, central nervous system; PACAP, pituitary adenylate cyclase activating polypeptide; VA, vasoactive intestinal peptide antagonist; VIP, vasoactive intestinal peptide.

The Journal of Clinical Investigation, Inc.

Volume 94, November 1994, 2020-2027 regulating central nervous system $(\mathrm{CNS})^{1}$ ontogenesis. Although environmental factors have been associated with microcephalies, (1) no etiological factor has been unequivocally identified. Furthermore, information on the ontogenetic events and molecular mechanisms involved in the pathology of CNS growth retardation is sparse. Recent reports have shown that inhibition of insulin-like growth factor- 2 activity induced a reduction in brain size. However, insulin-like growth factor-2 blockade produced a proportional reduction in both brain and body, (2) suggesting that microcephaly involves interference with other, more specific, growth factors. The most extensively studied experimental model for microcephaly is based on administration of the alkylating agent, methylazoxymethanol to pregnant rats (3-5). Prenatal treatment with this short-acting antimitotic agent produces severe microcephaly and behavioral deficits in newborn rats. Other substances that have been studied as inducers of microcephaly and neuroanatomical deficits include methylnitrosourea (6) and ochratoxin A (7).

A new model for microcephaly is now proposed based on the inhibition of the mitogenic and/or survival-promoting actions of vasoactive intestinal peptide (VIP) $(8-11)$. This study is the first to describe a microcephalic outcome produced by the inhibition of a specific neurochemical: VIP. The growth retardation was produced using a specific VIP antagonist (VA, neurotensin-VIP 7-28 hybrid antagonist) (12-14).

This work had its foundation in previous observations indicating that VIP has unique neurotrophic properties as demonstrated in primary cultures from the central nervous system. Subnanomolar amounts of VIP have been shown to increase the survival of spinal cord neurons and blockade of endogenous VIP by specific antiserum or VIP antagonists has produced neuronal cell death $(8,12)$. These survival-promoting effects were shown to be mediated by secondary molecules released from glia $(9,15)$. Subsequent reports have indicated that VIP can promote mitosis of astroglia (9) and neuroblasts from sympathetic ganglia (11). These in vitro studies were extended recently to whole embryo cultures (10). A 4-h treatment with VIP $\left(10^{-10} \mathrm{M}\right.$ to $\left.10^{-7} \mathrm{M}\right)$ was shown to dramatically stimulate growth of embryonic mice at gestational day 9.5 (E 9.5). VIP caused a concentration-dependent increase in somitic number, a marker of growth and maturation. An average of 5.2 new somites were produced with $10^{-7} \mathrm{M}$ VIP, while control embryos acquired an average of 2.2 new somites during the treatment period. Similar VIP growth-stimulating effects (11-63\% of increase from control) were evident on embryonic volume. In comparison to control embryos in culture, VIP increased the DNA and protein content by 103 and 63\%, respectively, indicating that VIP induced growth through large increases in mitotic 
activity and/or in survival of dividing precursors. When cells were labeled in S-phase with bromodeoxyuridine, it became evident that VIP produced a concentration-dependent increase in the number of cells in S-phase (up to a 5-6-fold increase) in both neuronal and nonneural tissues. At this stage, virtually all of the detectable VIP binding sites were confined to the central nervous system $(10,16)$ and addition of VIP to the culture medium produced a concentration-dependent decrease in binding sites. Addition of VA partially blocked both the VIPstimulated increases in embryonic growth and the VIP-induced down-regulation of VIP binding sites (10). Together these data suggest that during the period between E9 (closure of neural tube) and E12 (onset of neocortical neuronal migration), VIP regulates embryo growth through VIP binding sites localized to the CNS. At this time, the embryo may be vulnerable to pharmacological blockade of VIP actions that have consequences to brain development. This is also the period in which the production of precursor cells for the whole brain occurs, as does the commitment of cells to the glial lineage (17). With this critical period as a focus, the purpose of the present work was to assess the role of VIP on early CNS growth by injecting pregnant mice with an antagonist to VIP.

\section{Methods}

VA-hybrid VIP antagonist. To assess the role of VIP on early CNS growth a VIP antagonist was used. The pharmacological specificity of the VA has been investigated (12-14). VA is a hybrid peptide consisting of a carboxy fragment of VIP (VIP 7-28) and a six-amino acid fragment of neurotensin (13). This antagonist was found to have no agonist activity and to recognize both high and low affinity VIP binding sites on astroglia (12). At the lower affinity site associated with the generation of cAMP, VA exhibited 10-fold greater potency than VIP in displacing radiolabeled VIP from cortical astrocytes. At the high affinity site, VIP and VA were equipotent in binding displacement studies. The VIP antagonist exhibited tissue specificity as it did not block VIP receptors on lymphocytes (14). In contrast, VA inhibited VIP-stimulated proliferation and growth of lung cancer cells (18). Previous studies have shown that VA treatment of CNS cultures produced a $30 \%$ loss in the number of neurons (12). Administration of VA to neonatal animals caused delays in the acquisition of developmental milestones and damage to cerebral cortical neurons $(19,20)$. Importantly, co-treatment with neurotensin did not influence the biological activity associated with VA (19).

Drug administration. Pregnant NIH Swiss mice were injected intraperitoneally twice daily (8-9 AM and 6-7 PM) between E9.3 and E11.8 with $200 \mu \mathrm{l}$ of phosphate buffered saline (PBS) containing VA (synthesized as described previously) (13) or various neuropeptides purchased from Peninsula Laboratories Inc. (Belmont, CA) dissolved in $0.02 \%$ acetic acid. Experimental groups were as follows: $0.02,0.2$, or $2 \mu \mathrm{g} / \mathrm{g}$ body weight of VA; $0.002,0.02,0.2$, or $2 \mu \mathrm{g} / \mathrm{g}$ of VIP; $2 \mu \mathrm{g} / \mathrm{g}$ secretin; co-treatment with $2 \mu \mathrm{g} / \mathrm{g}$ of VIP and $0.2 \mu \mathrm{g} / \mathrm{g} \mathrm{VA}$; co-treatment with $2 \mu \mathrm{g} / \mathrm{g}$ pituitary adenylate cyclase activating peptide 1-38 (PACAP $1-38$ ), a recently discovered VIP-like peptide (21) and $0.2 \mu \mathrm{g} / \mathrm{g} \mathrm{VA}$; $2 \mu \mathrm{g} / \mathrm{g}$ PACAP 6-27 (a partial PACAP antagonist) (22), and PBS containing $0.02 \%$ acetic acid. At E9.8 and E11.8, one hour after the last injection, and at E17.8, mothers were sacrificed and the embryos removed. E17.8 was chosen as a termination point since at this gestational age the cortical plate is formed and gliogenesis is occurring in the germinal zone. To investigate the range of ontogenic vulnerability, administration of VA from E9.3 to E9.8, E11.8 or E17.8 was compared. In addition, in a separate group of animals, $2 \mu \mathrm{g} / \mathrm{g}$ VA or PBS was injected twice daily from E9.3 to E17.8, E12.3 to E14.8, or E15.3 to E17.8 to compare effects of VA before and during neuronal migration. At indicated ages, embryos were isolated and either frozen at $-80^{\circ} \mathrm{C}$ for biochemical analysis (DNA, protein and receptor assays) or fixed in $70 \%$ ethanol and paraffin embedded for mitosis evaluation by bromodeoxyuridine (BRDU) immunocytochemistry.

Biochemical analysis of embryos. As separate tissues, the head and the body were homogenized in $0.1 \mathrm{M}$ perchloric acid. The DNA content was measured as previously described $(23,24)$ and the protein content was measured using the protein analysis kit from Bio-Rad Laboratories (Hercules, CA) (25). All statistical analyses were performed by analysis of variance with Student-Newman-Keuls multiple comparison of means test. 6-28 embryos from at least three mothers were used in each experimental group. Values were obtained from three or more experiments.

Immunocytochemistry. To test the effects of VA treatment from E9.3 to E11.8 on mitosis, BRDU, a thymidine analog integrated in S-phase cells (26), was incorporated and detected as described (27). $20 \mathrm{~min}$ before sacrifice, $50 \mathrm{mg} / \mathrm{kg}$ BRDU, purchased from Sigma Chemical Co. (St. Louis, MO) in $200 \mu \mathrm{l}$ PBS was injected intraperitoneally to pregnant mice. Embryos were sacrificed at E9.8, E11.8, or E17.8, and fixed in $70 \%$ ethanol and paraffin embedded. Sagittal $5-\mu \mathrm{m}$ sections were cut and stained with hematoxylin and eosin or used for immunocytochemistry to detect nuclei which had incorporated BRDU. The proportion of BRDUlabeled cells was compared at the level of the prosencephalon (E9.8 and E11.8 embryos) or neocortical and cerebellar germinal zones (E17.8 embryos) and, in the body, at the level of somites (E9.8) or limb buds (E11.8). Cell counts were performed in four embryos per experimental group. Five serial sections were taken at $10-\mu \mathrm{m}$ intervals per embryo. On each section, at least 200 cells were counted for each area studied. In each group, embryos from at least two different pregnant mice treated in two experiments were included.

To assess effects on glia, immunocytochemistry with the glial marker RC2 (28) was performed on frozen sections. Embryos were sacrificed on E9.8 or E11.8 and then frozen by immersion in liquid nitrogen and processed for immunocytochemistry as previously described $(17,28,29)$.

VIP binding analyses. To evaluate the effects of VA treatment on VIP binding sites, in vitro autoradiography was performed on treated embryos. Embryos were rapidly frozen at $-80^{\circ} \mathrm{C}$ and $20-\mu \mathrm{m}$ cryostat sections were processed for binding with ${ }^{125} \mathrm{I}$-VIP as previously described(30). Sections were incubated in ${ }^{125}$ I-VIP, purchased from Amersham Corporation (Arlington Heights, IL), with and without $1 \mu \mathrm{M}$ VIP. The density of labeled VIP in various regions of the embryonic neural tube was analyzed by digitizing the film images using a MacintoshII-based image analysis system (IMAGE, Wayne Rasband, Research Services Branch, NIMH). Specific binding was determined by subtracting the light transmittance from brain sections incubated with $1 \mu \mathrm{M}$ unlabeled VIP from the total light transmittance. Measurements were made at the level of the prosencephalon at E9.8. The results were expressed as optical density units. In each experimental group, six embryos from three different pregnant mice treated in two different experiments were included.

\section{Results}

Biochemical analysis of embryos. Treatment with the VIP antagonist from E9.3 to E11.8 induced a dose-dependent decrease in the content of DNA (52\% of controls) and protein (43\% of controls) in the heads of E11.8 embryos (Fig. 1, $A$ and $B$ ). In contrast, the DNA and protein content of the body was less affected ( 89 and $72 \%$ of controls, respectively). Using the same VA treatment paradigm, animals that were sacrificed later at E17.8 had brain weights and size (59\% of controls, Figs. $1 C$ and $2 A$ ) that were reduced in a similar proportion to those from animals taken at E11.8; the weight loss was less marked in the body ( $82 \%$ of controls). Increased duration of VA treatment from E9.3 to E17.8 did not significantly change the extent of 

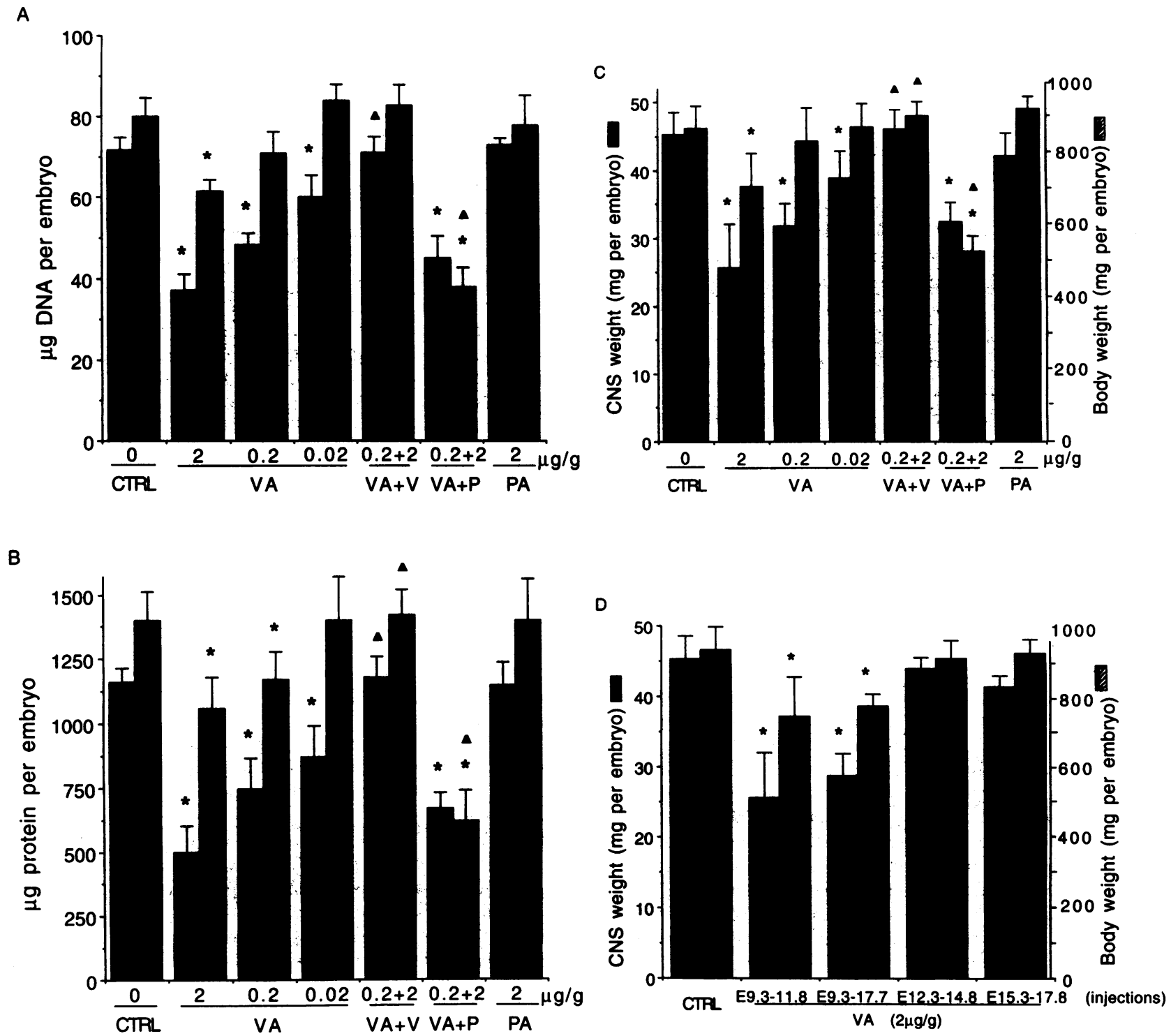

Figure 1. VA treatment produced a stage-dependent microcephaly. $(A)$ DNA content of E11.8 embryos $(n=7-12)$. (B) Protein content of E11.8 embryos $(n=8-12)$. (C) Weights of E17.8 embryos injected between E9.3 and E11.8 $(n=7-24)$. $(D)$ Weights of E17.8 embryos injected during different developmental periods $(n=6-24)$. Solid bars, head $(A$ and $B)$ or brain $(C$ and $D)$. Hatched bars, body. $C T R L$, control; VA, VIP antagonist; $V$, VIP; $P$, PACAP 1-38; PA, PACAP 6-27. Results are expressed as means \pm standard deviation; $\left(^{*}\right)$ differs from control $P<0.05 ;(\Delta)$ differs from the group treated with $0.2 \mu \mathrm{g}$ VA $(P<0.05)$.

microcephaly from that obtained from E9.3 to E11.8 (Fig. 1 D). Compared with controls, no significant reduction in brain size was observed in the embryos of mice injected with VA between E12.3 and 14.8 or E15.3 and 17.8 (Fig. $1 \mathrm{D}$ ). When dams were allowed to deliver, VA-treated pups were born alive at term. A related peptide, secretin, had no effect on the parameters under study (not shown). Co-treatment with a ten-fold molar excess of VIP abolished the VA-induced impairment of CNS and body growth (Figs. 1 and $2 \mathrm{~A}$ ). Co-treatment with the VIPlike peptide, PACAP, did not prevent the impairment of growth produced by VA (Figs. 1 and $2 A$ ). However, PACAP co-treatment resulted in greater decreases in body DNA, protein, and weight than were produced by VA alone (Fig. 1). Furthermore, PACAP 6-27, a putative PACAP antagonist (22), failed to inhibit embryonic growth (Fig. 1). Treatment of the dams with
VIP alone was toxic ( 0.002 to $0.02 \mu \mathrm{g} / \mathrm{g}$ ) or lethal ( 0.2 to $2 \mu \mathrm{g} /$ g) to the embryos (not shown).

Histological and immunocytochemical analyses. Compared with control, VA treatment resulted in a reduction in the thickness of the cortical wall (Fig. 2, $B$ and $C$ ). The thickness reduction affected the germinal and intermediate (prospective white matter) zones, but spared the cortical plate itself. In all treated embryos, the lower half of the intermediate zone was partially depopulated when compared to controls. No detectable histological lesions were observed in VA-treated tissue. The parallel reduction in DNA and protein content as well as normal cytoarchitecture of VA-treated embryos suggested a decreased cell number induced by mitotic inhibition and/or increased death of mitotic precursor cells. To test this hypothesis, BRDU was injected into pregnant dams $20 \mathrm{~min}$ before sacrifice. In E9.8 em- 

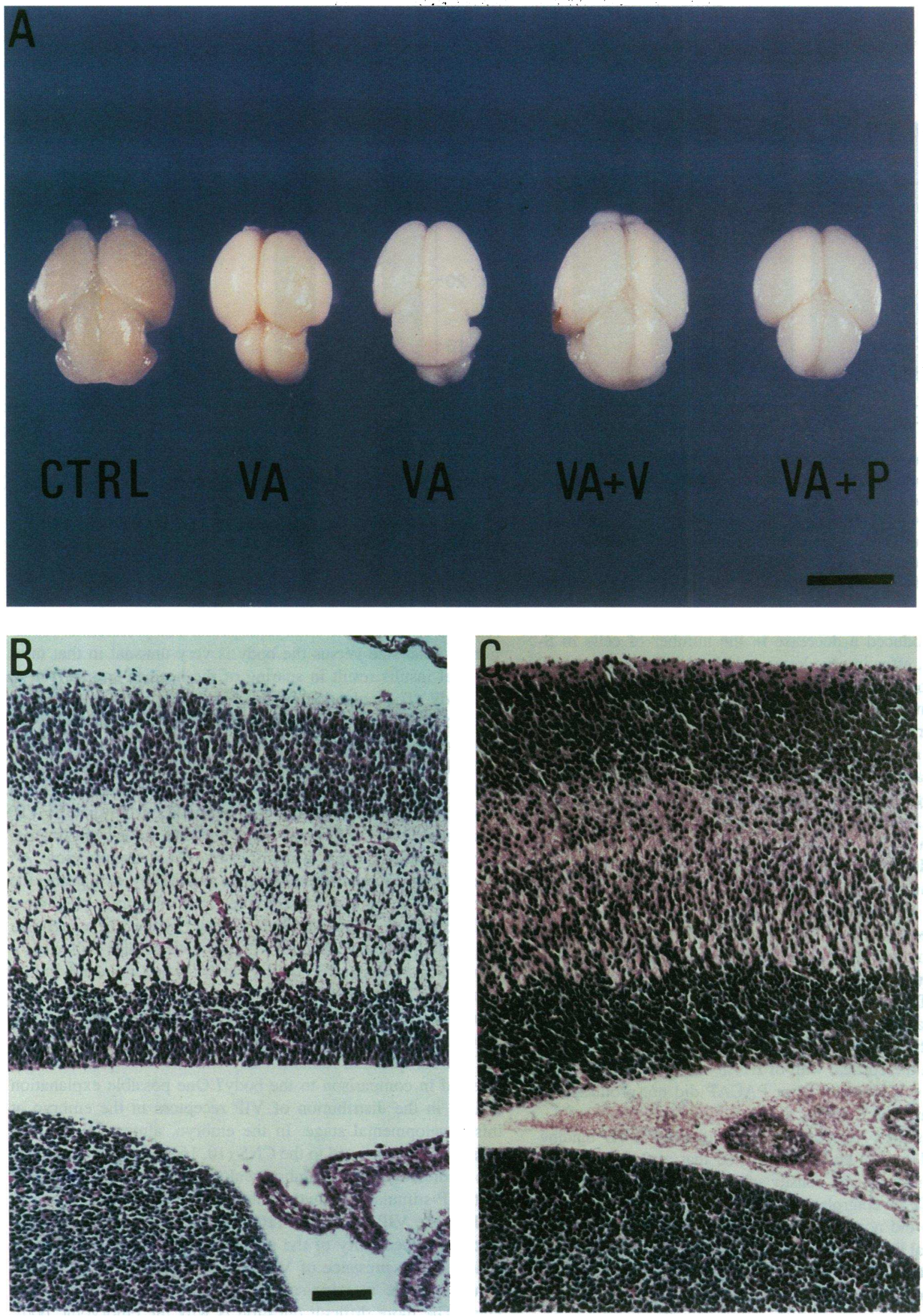

Figure 2. VA-inhibited brain growth without producing detectable gross or histological lesions. (A) Representative macroscopic appearance of E17.8 embryo brain treated between E9.3 and E11.8 with PBS alone (CTRL), $2 \mu \mathrm{g} / \mathrm{g}$ VA $(V A), 0.2 \mu \mathrm{g} / \mathrm{g}$ VA $(V A), 0.2 \mu \mathrm{g} / \mathrm{g}$ VA plus $2 \mu \mathrm{g} / \mathrm{g}$ VIP (VA + $V)$, and $0.2 \mu \mathrm{g} / \mathrm{g}$ VA plus $2 \mu \mathrm{g} / \mathrm{g}$ PACAP 1-38 $(V A+P)$. Bar, $0.5 \mathrm{~cm}$. (B) Histological appearance of neocortex from E17.8 embryo treated with $2 \mu \mathrm{g} / \mathrm{g}$ VA between E9.3 and E11.8. Some variation in the cellular density in the cortical plate was observed in both control and treated embryos and was probably due to irregularities in section thickness. $(C)$ Neocortex from E17.8 embryo treated with PBS alone. Tissue stained with hematoxylin and eosin. 


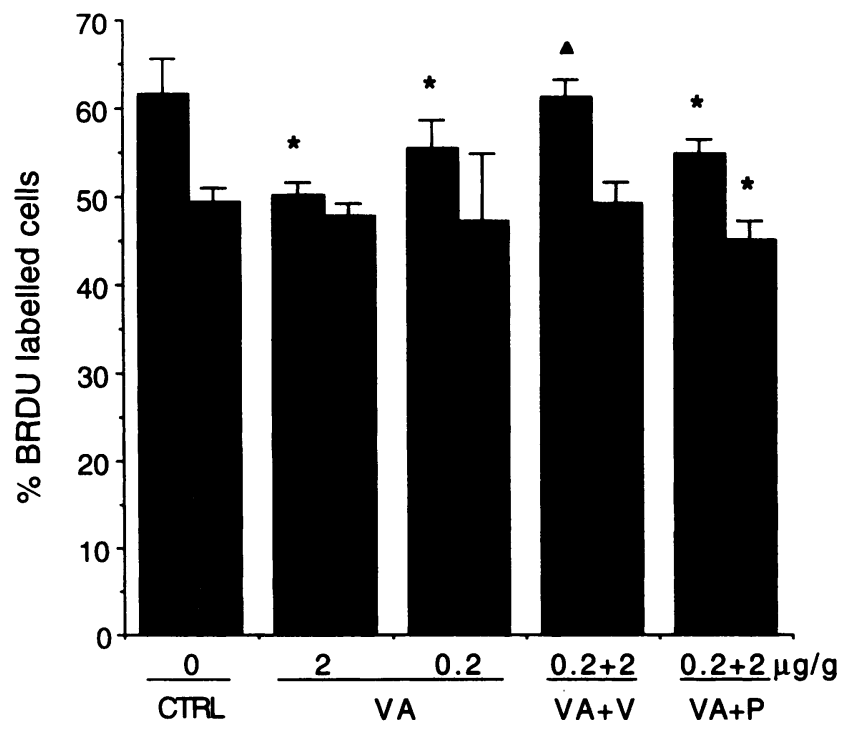

Figure 3. VA-inhibited mitotic activity and/or cell survival of neuronal cells. The proportion of BRDU labeled cells in the CNS (solid bars) and body (hatched bars) of E9.8 embryos $(n=20)$ treated with VA, VA and VIP $(V A+V)$, or VA and PACAP 1-38 $(V A+P)$ was shown in comparison with data obtained in controls $(C T R L)$. Results are expressed as means \pm standard deviation; $\left({ }^{*}\right)$ different from control $(P$

$<0.05)$; ( $\Delta)$ differs from the group treated with $0.2 \mu \mathrm{g} / \mathrm{g} \mathrm{VA}(P<0.05)$.

bryos, VA produced a decrease in the number of cells in Sphase (Fig. 3), affecting predominantly neural ( $82 \%$ of control) rather than non-neural ( $96 \%$ of control) tissues. Co-treatment with VIP but not PACAP, prevented the VA-mediated decrease in the number of BRDU-positive cells (Fig. 3). Co-treatment with PACAP resulted in a reduction in cells in S-phase in the body which was not observed in embryos treated with VA alone.

To investigate the range of ontogenic vulnerability of these effects, BRDU incorporation was compared in embryos treated with VA from E9.3 to either E9.8, E11.8 or E17.8. In contrast to the VA-induced reduction of BRDU labeled cells observed at E9.8, the number of S-phase cells in the E11.8 and E17.8 embryos was not significantly changed from that of control (data not shown).

The glial marker, RC2, was used to determine if antagonist treatment altered glial morphology. The intensity of labeling, the density of labeled glial fibers, the radial distribution and fascicular organization were not modified by VA treatment in the neuroepithelium of E9.8 and E11.8 embryos (data not shown) and in the cortical wall of E17.8 embryos. Secretin and co-treatment of VA with VIP or PACAP did not produce any detectable change in the RC2 staining.

VIP binding sites. Autoradiographic studies of ${ }^{125} \mathrm{I}-\mathrm{VIP}$ binding on treated and control embryos revealed that at $2 \mu \mathrm{g} / \mathrm{g}$ of VA elicited an increase in the density of VIP binding sites in E9.8 embryos (136\% of controls) (Fig. 4). A lower concentration of VA $(0.2 \mu \mathrm{g} / \mathrm{g})$ did not reveal a significant increase VIP binding sites. The partial PACAP antagonist (PACAP 6-27) had no effect. VIP binding sites were present in the germinal neuroepithelium at E9.8 (Fig. 4) but were reduced by E11.8 (data not shown).

\section{Discussion}

This study shows that blocking the functions of VIP or a VIPlike molecule for a brief period during early organogenesis

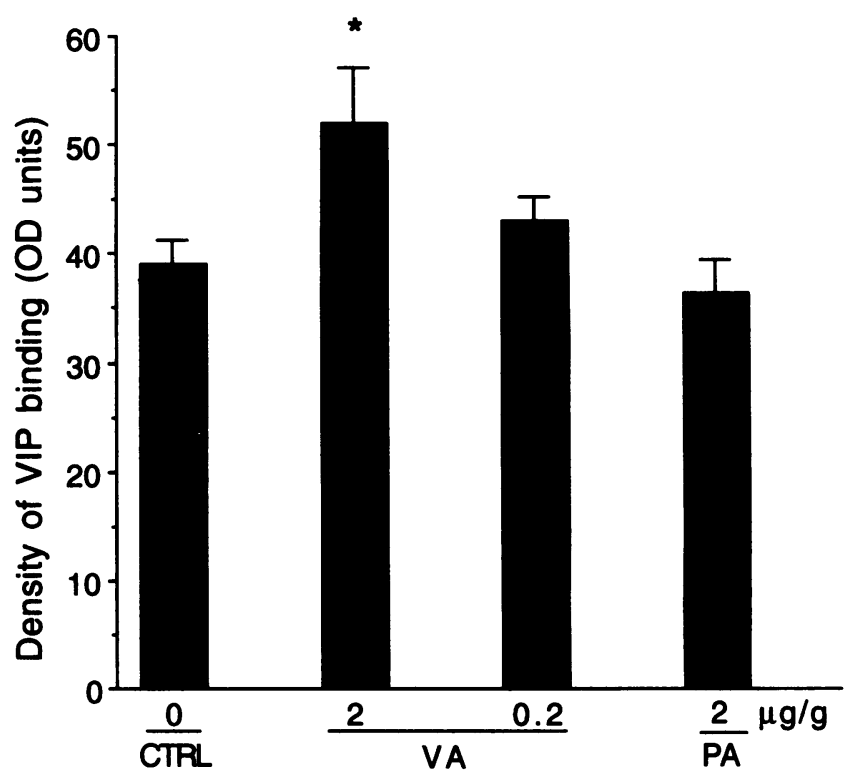

Figure 4. VA-increased ${ }^{125}$ I-VIP binding in E9.8 embryos $(n=6)$. CTRL, control; VA, VIP antagonist; PA, PACAP 6-27. Results are expressed as means \pm standard deviation. $\left({ }^{*}\right)$ Differs from control $(P$ $<0.05)$.

retarded growth of the embryonic brain. The preferential reduction in head size versus the body is very unusual in that most prenatal insults result in sparing of the head in comparison to the body. The reduction of head size, we believe, is due to both the remarkable specificity of the VIP antagonist and the fundamental regulatory influence of VIP on mitogenic events in the brain during the critical period when neural tube closure and associated processes are occurring. These studies clearly show that mitogenic activity was inhibited by the specific VIP antagonist at this embryonic stage and that the growth retardation was severe, even discernible by visual examination. An alternate or co-existing mechanism potentially explaining the microcephalic brains is a selective death of mitotic precursors in the primitive neuroepithelium induced by the VIP antagonist. This hypothesis is consistent with previous studies indicating a survival-promoting effect of VIP $(8,9,15)$ and cell death induction by the VIP antagonist (12). Death rather than the inhibition of mitosis in precursor cells during this critical period could account for the loss of cells later in gestation.

But the question remains: why was the head preferentially affected in comparison to the body? One possible explanation resides in the distribution of VIP receptors in the embryo at this developmental stage. In the embryo, almost all the VIP receptors are restricted to the CNS $(10,16)$. Furthermore, previous studies with whole embryo cultures at E9.5 have indicated that VIP-stimulated growth in the brain was completely inhibited by the VIP antagonist (10). These data strongly indicate that the vulnerability of the embryonic CNS to VIP blockade resides in the presence of VIP receptors playing an important regulatory role in stage-specific mitogenesis and/or cell survival. The more difficult question is why the embryonic body was decreased $(<10 \%)$ by so little in comparison to the brain losses (50\%) in DNA, protein and weight. Two previous observations made on whole embryo cultures may contribute to our 
understanding of why body growth was less retarded than brain growth after maternal VA treatment. Despite the absence of detectable VIP binding sites in the body, treatment of a E8.5 embryo in vitro with VIP resulted in a dramatic growth of both the brain and the body (10). Therefore, at some level, the body was capable of responding to a VIP-mediated stimulus. What is not yet clear, is whether the growth of the body is due to: (a) the stimulation of low abundance, undetectable VIP receptors or, $(b)$ a response of the body to secondary molecules elicited by VIP stimulation of CNS receptors. If the low level receptor hypothesis is true, then the lack of response of the body to the VIP antagonist could be explained by the known preference of the VIP antagonist for CNS receptors versus those in the periphery (14). The tissue specificity of the antagonist may account for the observed preferential loss in the head in comparison to the body. Alternatively, if it is true that VIP receptors are restricted to the CNS at this stage in development, then a two-receptor hypothesis must be invoked to explain the observed effects: one receptor that regulates CNS mitosis and/ or cell survival of dividing cells in the neuroepithelium, and another receptor that regulates the secretion of secondary factor(s) that control body growth. As with the first hypothesis, the lower response of the body would be attributed to a reduced ability of the antagonist to block the second class of VIP receptors regulating body growth.

The molecular identity of the receptor(s) mediating the growth-promoting actions of VIP is not yet clear. However, cDNA's encoding rat (31) and a similar human (32) form of the VIP receptor recently have been cloned. This receptor was detected in lung, liver and intestine as well as in the cortex, hippocampus, hypothalamus, and cerebellum of the brain. A second, high affinity receptor for VIP has been cloned which was enriched in rat pituitary, olfactory bulb, thalamus, hippocampus, and the suprachiasmatic nucleus (33). The two VIP receptors were found to be pharmacologically distinct and had a 50\% identity at the amino acid level. Similarities were greatest in the putative transmembrane domains. A similarity in transmembrane domains was also found between the two VIP receptors and the PACAP family of receptors, which consists of five receptors derived from the same gene by alternative splicing $(34,35)$. These PACAP receptors have different affinities for PACAP 38, PACAP 27, and VIP and in several tissues including lung, liver, and intestines a shared PACAP/VIP receptor has been identified. However, specific high affinity PACAP receptors have been identified in the brain which do not recognize VIP (36). The failure of PACAP to act like VIP and prevent VA-mediated inhibition of growth and development suggests that PACAP was unable to act at those receptor sites at which VA inhibited growth and development. Previous studies of the mitogenic properties of low concentrations $(<100 \mathrm{pM})$ of VIP on astroglia suggested that the receptor mediating these events was not linked to adenylate cyclase (9); rather, a calcium mobilizing mechanism was most likely associated with both the secretory and mitogenic actions of VIP (37). When the expression and signal transduction pathways of the known VIP and PACAP receptors have been characterized in the embryonic CNS, the identity of the growth-related receptor(s) may become apparent.

The previous discussion presumed that VIP was the endogenous ligand that was blocked by administration of the hybrid peptide antagonist. Certainly, the fact that the antagonist has a $80 \%$ identity with VIP (amino acids 7-28) supports the conclu- sion that VIP is the natural ligand for this drug. Furthermore, in vitro studies $(12,14)$ and in vivo studies $(13,19)$ have demonstrated specificity. In the current study, co-treatment with a 10fold molar excess of VIP (and not with VIP-related peptides) abolished the VA-induced impairment of CNS and body growth (Fig. 1 and 2), demonstrating the specificity of the observed effects. However, these results could have been achieved if the antagonist was blocking some other VIP-like substance. To address this possibility, a series of experiments were designed to test whether PACAP might play such a role. However, cotreatment of the VIP antagonist with PACAP did not prevent the impairment of growth produced by VA (Fig. 1 and $2 A$ ). Furthermore, PACAP 6-27, a partial PACAP antagonist (22), had no effect on embryonic growth (Fig. 1), suggesting that VA inhibits a specific VIP function not shared by PACAP. Although co-treatment with PACAP had no effect on the VAinduced reduction of cells in S-phase in the CNS, it resulted in a decrease of BRDU labeling in the body not observed in embryos treated with VA alone (Fig. 3). This suggests that PACAP, at this concentration, had an effect on cell number opposite to that reported for VIP. Thus, based on these experiments, the most likely explanation of the data was that the hybrid antagonist was not only specifically blocking the effects of VIP, but also acting at a VIP receptor which was not stimulated/recognized by PACAP.

The radial glial fascicles and their affiliated migrating neurons constitute an ontogenic unit organizing the developing cerebral cortex $(38,39)$. The extent of the cortical surface is determined by the number of these adjacent neuronal-glial modules. These units are present in the primitive neuroepithelium soon after neural tube closure, much earlier than the initiation of neuronal migration (17). In VA-treated animals, the integrity of the neuronal-glial unit was preserved, as demonstrated by the normal glial organization and density and by the conserved cortical plate cytoarchitecture and thickness. In contrast, the cortical surface was reduced, strongly suggesting a diminished number of neuronal-glial modules formed in VA-treated embryos. Three possibilities have been considered: $(a)$ the mitotic inhibition and/or cell death induced by the VA in the primitive germinal zone primarily affected the glial precursors with a secondary inhibition of neuronal production due to lower amounts of neurotrophic factors secreted by glial cells; $(b)$ the reverse may also occur; i.e., the neuronal precursors may be reduced with a secondary effect on glia production; $(c)$ both neuronal and glial populations could be affected by VA.

Treatment with the VIP antagonist resulted in a reduction of the intermediate zone of the cortical wall. This loss might reflect a decreased neuronal migration due to a reduced number of neuronal precursors in the germinative zone.

VIP antagonist treatment of mid-gestation mice resulted in an increase in the number of VIP binding sites observed in the CNS of the embryos. Additionally, in previous studies we showed that VIP treatment decreased VIP binding in both the neonatal (20) and embryonic (10) brain and that VA treatment increased VIP binding (10). Our explanation of this response was that the VIP antagonist-induced an upregulation of VIP receptors. This increase in VIP binding sites strongly suggests that the antagonist reached CNS receptors in the present study and that the increase was a supersensitivity pharmacological response attributable to chronic blockade of the VIP receptors. These findings indicate that the effects of the VIP antagonist 
on embryonic growth were due to actions on VIP receptors in the embryonic CNS. As emphasized previously, the lack of any apparent growth in response to PACAP suggests that those receptors which can be occupied by both VIP and PACAP were probably not involved in VIP-mediated increases in mitogenesis.

The microcephaly produced by the VIP antagonist showed striking age dependency. In contrast to the mitotic inhibitory effects of VA observed at E9.8, the number of S-phase cells in the E11.8 and E17.8 embryos was not significantly changed from that of control (data not shown). In addition, the embryos treated between E12.3-14.8 and E15.3-17.8 did not display growth retardation of either CNS or body. These data suggest that the VIP regulation of cell division was stage specific as it was limited to the developmental period between E9 and E11, in agreement with previous in vitro results (10). The transient presence of VIP receptors on germinal neuroepithelium may explain this marked stage dependency to the vulnerability to VA. In addition, the marked developmental sensitivity argues against the VIP antagonist producing indirect effects on the fetus through hemodynamic, ischemic, and/or possible toxic effects on maternal systems. Alternatively, the stage dependency of the VIP antagonist effects on brain growth might be related to a reduced ability of the antagonist to reach the embryonic brain at later stages, making it necessary to use higher doses of VA to elicit changes in older brains. However, radiolabeled VA has been found to reach the brains of E16 to E18 rat fetuses after either intraperitoneal $(0.005 \%$ reached the fetal brain) or intrauterine $(0.01 \%$ reached the fetal brain) treatment of the mother (I. Gozes, unpublished results). These data suggest that VA was accessible to the fetal brain of the mouse from E11 to E17, but did not have a growth-inhibiting effect.

The present study has shown that administration of a VIP antagonist to a mid-gestational mouse produced a severe microcephaly in the fetus. This effect is believed to be associated with inhibition of mitosis and/or decreased cell survival of dividing precursors in the primitive neuroepithelium during a critical period in development. This in vivo evidence underscores the importance of VIP in the normal growth and development of the embryo and also furnishes a model for microcephaly. These data imply that any interference, whether pharmacological, viral, or nutritional, with VIP action at this critical period in development may result in reduced brain size. Our hope is that an understanding of VIP action during pregnancy may provide insight into formulating a more unifying hypothesis for microcephaly in children.

\section{Acknowledgments}

We thank Drs. H. Def. Webster and P. G. Nelson for their support. The technical support of S. Rubinrout, H. Leininger, E. Abebe, and R. Riggs is gratefully acknowledged.

This work was supported in part by the US-Israel Binational Science Foundation.

\section{References}

1. Opitz, J. M., and M. C. Holt. 1990. Microcephaly: general considerations aids to nosology. J. Craniofacial Genet. Dev. Biol. 10:175-204.

2. DeChiara, T. M., A. Efstratiadis, and E. J. Robertson. 1990. A growthdeficiency phenotype in heterozygous mice carrying an insulin-like growth factor gene disrupted by targeting. Nature (Lond.). 345:78-80.
3. Banfi, S., L. Dorigotti, M. P. Abbracchio, W. Balduini, E. Coen, C. Ragusa, and F. Cattabeni. 1984. Methylazoxymethanol microencephaly in rats: neurochemical characterization and behavioral studies with the nootropic oxiracetam. Pharmacol. Res. Commun. 16:67-83.

4. Mercugliano, M., S. L. Hyman, and M. L. Batshaw. 1990. Behavioral deficits in rats with minimal cortical hypoplasia induced by methylazoxymethanol acetate. Pediatrics. 85:432-436.

5. Ashwell, K. W., and W. S. Webster. 1988. The contribution of primary and secondary neuronal degeneration to prenatally-induced micrencephaly. Neurotoxicol. Teratol. 10:65-73.

6. Hashimoto, Y., and M. Mizutani. 1991. Early neurobehavioral disorders in the micrencephalic offspring induced by prenatal treatment with $\mathrm{N}$-methyl-Nnitrosourea. J. Vet. Med. Sci. 53:643-649.

7. Fukui, Y., S. Hayasaka, M. Itoh, and Y. Takeuchi. 1992. Development of neurons and synapses in ochratoxin A-induced microcephalic mice: a quantitative assessment of somatosensory cortex. Neurotoxicol. Teratol. 14:191-196.

8. Brenneman, D. E., and L. E. Eiden. 1986. Vasoactive intestinal peptide and electrical activity influence neuronal survival. Proc. Natl. Acad. Sci. USA. 83:1159-1162.

9. Brenneman, D. E., T. Nicol, D. Warren, and L. M. Bowers. 1990. Vasoactive intestinal peptide: a neurotrophic releasing agent and an astroglia mitogen. $J$. Neurosci. Res. 25:386-394.

10. Gressens, P., J. M. Hill, I. Gozes, M. Fridkin, and D. E. Brenneman. 1993. Growth factor function of vasoactive intestinal peptide in whole cultured mouse embryos. Nature (Lond.). 362:155-158.

11. Pincus, D. W., E. M. DiCicco-Bloom, and I. B. Black. 1990. Vasoactive intestinal peptide regulates mitosis, differentiation and survival of cultured sympathetic neuroblasts. Nature (Lond.). 343:564-567.

12. Gozes, I., S. K. McCune, L. Jacobson, D. Warren, T. W. Moody, M. Fridkin, and D. E. Brenneman. 1991. An antagonist to vasoactive intestinal peptide affects cellular functions in the central nervous system. J. Pharmacol. Exp. Ther. 257:959-966.

13. Gozes, I., E. Meltzer, S. Rubinrout, and D. E. Brenneman. 1989. Vasoactive intestinal peptide potentiates sexual behavior: Inhibition by a novel antagonist. Endocrinology. 125:2945-2949.

14. Gozes, Y., D. E. Brenneman, M. Fridkin, R. Asofsky, and I. Gozes. 1991. A VIP antagonist distinguishes VIP receptors in spinal cord cells and lymphocytes. Brain Res. 540:19-321.

15. Brenneman, D. E., E. A. Neale, G. A. Foster, S. W. d'Autremont, and G. L. Westbrook. 1987. Nonneuronal cells mediate neurotrophic action of vasoactive intestinal peptide. J. Cell Biol. 104:1603-1610.

16. Hill, J. M., D. V. Agoston, P. Gressens, and S. K. McCune. 1994. Patterns of VIP mRNA and VIP receptor distribution in the developing rat bran: Relation to ontogenetic events. J. Comp. Neurol. 342:186-205.

17. Gressens, P., F. Gofflot, G. Van Maele-Fabry, J. P. Misson, J. F. Gadisseux, P. Evrard, and J. J. Picard. 1992. Early neurogenesis and teratogenesis in whole mouse embryo cultures. Histochemical, immunocytological and ultrastructural study of premigratory neuronal-glial units in normal mouse embryos influenced by cocaine and retinoic acid. J. Neuropathol. \& Exp. Neurol. 51:206-219.

18. Moody, T. W., F. Zia, M. Draoui, D. E. Brenneman, M. Fridkin, A. Davidson, and I. Gozes. 1993. A vasoactive intestinal peptide antagonist inhibits non-small cell lung cancer growth. Proc. Natl. Acad. Sci. USA. 90:4345-4349.

19. Hill, J. M., I. Gozes, J. L. Hill, M. Fridkin, and D. E. Brenneman. 1991. Vasoactive intestinal peptide antagonist retards the development of neonatal behaviors in the rat. Peptides. 12:187-192.

20. Hill, J. M., R. F. Mervis, J. Politi, S. K. McCune, I. Gozes, M. Fridkin, and D. E. Brenneman. 1994. Blockade of VIP during neonatal development induces neuronal damage and increases VIP and VIP receptors in brain. Ann. N.Y. Acad. Sci. In press.

21. Miyata, A., L. Jiang, R. D. Dahl, C. Kitada, K. Kubo, M. Fujino, N. Minamino, and A. Arimura. 1990. Isolation of a neuropeptide corresponding to the $\mathrm{N}-20$ terminal 27 residues of the pituitary adenylate cyclase activating polypeptide with 38 residues (PACAP38). Biochem. Biophys. Res. Commun. 170:643648.

22. Robberecht, P., M. C. Woussen-Colle, P. De Neef, P. Gourlet, L. Buscail, A. Vandermeers, M. C. Vandermeers-Piret, and J. Christophe. 1991. The two forms of the pitutary adenylate cyclase activating polypeptide PACAP(1-27) and PACAP (1-38) interact with distinct receptors on rat pancreatic AR 4-2J cell membranes. FEBS (Fed. Eur. Biochem. Soc.) Lett. 286:133-136.

23. Burton, K. 1956. Study of the conditions and mechanism of the diphenylamine reaction for the colorimetric estimation of deoxyribonucleic acid. Biochem. J. 62:315-323.

24. Munro, H. N. 1968. The determination of nucleic acids. Methods Biochem. Anal. 14:113-176.

25. Bradford, M. 1976. A rapid and sensitive method for the quantitation of microgram quantities of protein utilizing the principle of protein-dye binding. Anal. Biochem. 72:248-254.

26. Gratzner, H. G. 1982. Monoclonal antibody to 5-bromo- and 5-iododeoxy- 
uridine: A new reagent for detection of DNA replication. Science (Wash. DC). 218:474-475.

27. Takahashi, T., R. S. Nowakowski, and V. S. Caviness. 1992. Cell cycle parameters and patterns of nuclear movement in the neocortical proliferative zone of the fetal mouse. J. Neurocytol. 21:185-197.

28. Misson, J. P., M. A. Edwards, M. Yammamoto, and V. S. Caviness. 1988. Identification of radial cells within the developing murine central nervous system: studies based upon a new immunocytochemical marker. Dev. Brain Res. 44:427431.

29. Gadisseux, J. F., P. Evrard, J. P. Misson, and V. S. Caviness. 1989. Dynamic structure of the radial glial fiber system of the developing murine cerebral wall: an immunocytochemical analysis. Dev. Brain Res. 50:55-67.

30. Hill, J. M., A. Harris, and D. I. Hilton-Clarke. 1992. Regional distribution of guanine nucleotide-sensitive and guanine nucleotide-insensitive vasoactive intestinal peptide receptors in rat brain. Neuroscience. 48:925-932.

31. Ishihara, T., R. Shigemoto, K. Mori, K. Takahashi, and S. Nagata. 1992. Functional expression and tissue distribution of a novel receptor for vasoactive intestinal peptide. Neuron. 8:811-819.

32. Sreedharan, S. P., D. R. Patel, J. X. Huang, and E. J. Goetzl. 1993. Cloning and functional expression of a human neuroendocrine vasoactive intestinal peptide. Biochem. Biophys. Res. Commun. 193:546-553.
33. Lutz, E. M., W. J. Sheward, K. M. West, J. A. Morrow, G. Fink, and A. J. Harmar. 1993. The VIP ${ }_{2}$ receptor: molecular characterization of a cDNA encoding a novel receptor for vasoactive intestinal peptide. FEBS (Fed. Eur. Biochem. Soc.) Lett. 334:3-8.

34. Pisegna, J. R., and S. A. Wank. 1993. Molecular cloning and functional expression of the pituitary adenylate cyclase activating polypeptide type I receptor. Proc. Natl. Acad. Sci. USA. 90:6345-6349.

35. Spengler, D., C. Waeber, C. Pnataloni, F. Holsober, J. Bockaert, P. H. Seeburg, and L. Journot. 1993. Differential signal transduction by five splice variants of the PACAP receptor. Nature (Lond.). 365:170-175.

36. Arimura, A. 1992. Receptors for pituitary adenylate cyclase-activating polypeptide: comparison with vasoactive intestinal peptide receptors. Trends Endrocrinol. Metab. 3:288-294.

37. Fatatis, A., L. A. Holtzclaw, R. Avidor, D. E. Brenneman, and J. T. Russell. 1994. Vasoactive intestinal peptide increases intracellular calcium in astroglia: synergism with alpha-adrenergic receptors. Proc. Natl. Acad. Sci. USA. 91:2036-2040.

38. Gadisseux, J. F., and P. H. Evrard. 1985. Glial-neuronal relationship in the developing central nervous system. Dev. Neurosci. 7:12-32.

39. Gressens, P., C. H. Richelme, H. J. Kadhim, J. F. Gadisseux, and P. H. Evrard. 1992. The germinative zone produces most cortical astrocytes after neuronal migration in developing mammalian brain. Biol. Neonat. 61:4-24. 